The INL is a

U.S. Department of Energy

National Laboratory

operated by

Battelle Energy Alliance

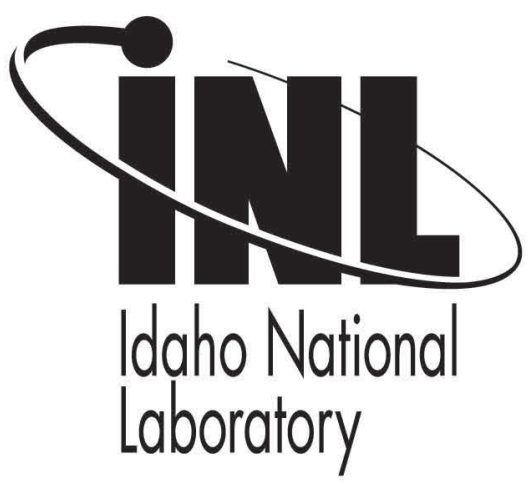

\title{
Passive Measurement of Organic-Scintillator Neutron Signatures for Nuclear Safeguards Applications
}

\section{IEEE Nuclear Science Symposium}

Jennifer L. Dolan

Eric C. Miller

Alexis C. Kaplan

Andreas Enqvist

Marek Flaska

Alice Tomanin

Paolo Peerani

David L. Chichester

Sara A. Pozzi

\section{October 2012}

This is a preprint of a paper intended for publication in a journal or proceedings. Since changes may be made before publication, this preprint should not be cited or reproduced without permission of the author. This document was prepared as an account of work sponsored by an agency of the United States Government. Neither the United States Government nor any agency thereof, or any of their employees, makes any warranty, expressed or implied, or assumes any legal liability or responsibility for any third party's use, or the results of such use, of any information, apparatus, product or process disclosed in this report, or represents that its use by such third party would not infringe privately owned rights. The views expressed in this paper are not necessarily those of the United States Government or the sponsoring agency. 


\title{
Passive Measurement of Organic-Scintillator Neutron Signatures for Nuclear Safeguards Applications
}

\author{
Jennifer L. Dolan, Member, IEEE, Eric C. Miller, Alexis C. Kaplan, Andreas Enqvist, Member, IEEE, Marek Flaska, \\ Member, IEEE, Alice Tomanin, Paolo Peerani, Member, IEEE, David L. Chichester, Member, IEEE, and Sara A. \\ Pozzi, Member, IEEE
}

\begin{abstract}
At nuclear facilities, domestically and internationally, most measurement systems used for nuclear materials' control and accountability rely on $\mathrm{He}-3$ detectors. Due to resource shortages, alternatives to $\mathrm{He}-3$ systems are needed. This paper presents preliminary simulation and experimental efforts to develop a fast-neutron-multiplicity counter based on liquid organic scintillators. This mission also provides the opportunity to broaden the capabilities of such safeguards measurement systems to improve current neutron-multiplicity techniques and expand the scope to encompass advanced nuclear fuels.
\end{abstract}

\section{INTRODUCTION}

$\mathrm{N}$ UCLEAR safeguards are defined as the efforts to prevent diversion of fissile material. Given the number of nuclear facilities and current proliferation threats across the world, new technologies are needed to maintain successful international safeguards efforts. Specifically, international communities' desired increase in fuel reprocessing warrants innovation in new safeguards techniques to minimize the associated nonproliferation risks. The International Atomic Energy Agency has asked for active research efforts in the development of new safeguards systems.

Many current safeguards measurement systems used at nuclear facilities, both domestically and internationally, rely on He-3 detectors and well established theory to interpret coincidence and multiplicity measurements for verifying quantities of special nuclear material (SNM). Due to resource shortages, alternatives to these existing He-3 based systems are being sought. Work is also underway to broaden the capabilities of these types of measurement systems in order to improve current multiplicity analysis techniques and to discover novel methods for nuclear material characterization. Organic scintillation detectors are a promising candidate for

Manuscript received on November 16, 2012. This research was partially funded by the National Science Foundation and the Domestic Nuclear Detection Office of the Department of Homeland Security through the Academic Research Initiative Award \# CMMI 0938909 and performed under the Nuclear Forensics Graduate Fellowship Program, which is sponsored by the U.S. Department of Homeland Security, Domestic Nuclear Detection Office and the U.S. Department of Defense, Defense Threat Reduction Agency.

J. L. Dolan, E. C. Miller, A. C. Kaplan, A. Enqvist, M. Flaska, and S. A. Pozzi are with the Nuclear Engineering and Radiological Sciences Department, University of Michigan, Ann Arbor, MI 48109, USA (telephone: 734-615-4970, e-mail: pozzisa@umich.edu).

A. Tomanin and P. Peerani are with the European Commission, Joint Research Centre, IPSC, Ispra 21020, Italy (e-mail: paolo.peerani@jrc.ec.europa.eu).

D. L. Chichester is with the Idaho National Laboratory, Idaho Falls, ID 83403, USA (e-mail: david.chichester@inl.gov). innovative neutron measurement systems to identify and characterize nuclear materials.

Passive measurements were performed on nuclear materials at the Joint Research Centre (JRC) in Ispra, Italy in April of 2012. These measurements allowed a survey of various neutron signatures available when measuring neutrons with liquid organic scintillators. A measurement system developed at the University of Michigan (UM) was used to measure wellcharacterized nuclear material. Analyses of the acquired data have led to a thorough understanding of potential data-analysis algorithms and techniques useful in advanced safeguards development. Additionally, this research strongly supports the importance of using of Monte Carlo simulations, specifically of the MCNPX-PoliMi code [1], in nuclear safeguards research and development through benchmarking exercises.

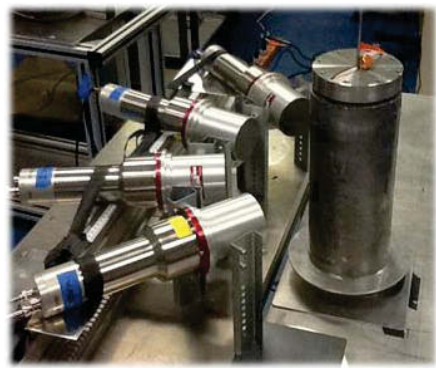

(a)

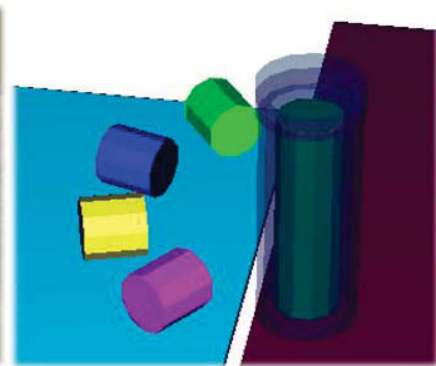

(b)
Fig. 1. Experimental (a) and simulated (b) configurations including liquid scintillators (Eljen Technology EJ-309) $20 \mathrm{~cm}$ from the center of the sample (MOX powder surrounded by $1 \mathrm{~cm}$ of lead).

\section{MEASURING FAST-NeUtRON MultiPlicity WITH LIQUID SCINTILLATORS}

Measurements were performed on fissile materials during the measurement campaign at the JRC's PERLA laboratory. A UM measurement system (Fig. 1a) was utilized and the results were then replicated with MCNPX-PoliMi. In addition, dataanalysis algorithms were tested for their potential and limitations. Comparing the measured and simulated results built confidence in the use of simulation and modeling tools to design fast-neutron-multiplicity systems. Combinations of $\mathrm{PuO}_{2}$ pellets as well as mixed-oxide (MOX) fuel samples were measured to asses a plutonium-mass sensitivity metric. The measured plutonium masses ranged from 20 to $60 \mathrm{~g}$ for the $\mathrm{PuO}_{2}$ pellets and 160 and $190 \mathrm{~g}$ for the MOX powder samples, details are outlined in Table 1 . The measured materials are similar to those measured in low-level plutonium-sample inventory counters and helped provide insight into how 
organic scintillators detectors would respond to such materials.

TABLE I. ISOTOPIC MASSES FOR THE MEASURED JRC PUO 2 PELLET COMBINATIONS AND MOX SAMPLES.

\begin{tabular}{cccccc} 
Isotope & $\begin{array}{c}\mathrm{PuO}_{2}-1 \\
(\mathrm{~g})\end{array}$ & $\begin{array}{c}\mathrm{PuO}_{2}-2 \\
(\mathrm{~g})\end{array}$ & $\begin{array}{c}\mathrm{PuO}_{2}-3 \\
(\mathrm{~g})\end{array}$ & $\begin{array}{c}\mathrm{MOX}-1 \\
(\mathrm{~g})\end{array}$ & $\begin{array}{c}\mathrm{MOX}-2 \\
(\mathrm{~g})\end{array}$ \\
\hline $\mathrm{U}-234$ & -- & -- & -- & 0.05 & 0.06 \\
$\mathrm{U}-235$ & -- & -- & -- & 4.79 & 5.50 \\
$\mathrm{U}-236$ & -- & -- & -- & 0.05 & 0.06 \\
$\mathrm{U}-238$ & -- & -- & -- & 670.50 & 769.48 \\
$\mathrm{Pu}-238$ & 0.02 & 0.03 & 0.04 & 0.24 & 0.27 \\
$\mathrm{Pu}-239$ & 14.24 & 39.66 & 52.33 & 111.81 & 127.02 \\
$\mathrm{Pu}-240$ & 5.39 & 9.31 & 11.26 & 47.00 & 53.39 \\
$\mathrm{Pu}-241$ & 0.13 & 0.23 & 0.27 & 1.67 & 1.90 \\
$\mathrm{Pu}-242$ & 0.29 & 0.37 & 0.41 & 3.38 & 3.84 \\
$\mathrm{Am}-241$ & 0.80 & 1.33 & 1.59 & 5.12 & 5.82 \\
$\mathrm{O}$ & 2.75 & 6.75 & 8.74 & 166.22 & 184.00 \\
Total & 23.63 & 57.68 & 74.65 & 1010.83 & 1151.33 \\
\hline
\end{tabular}

\section{A. Measurement System}

The UM measurement systems (Fig. 1a) consist of waveform digitizers with custom data-acquisition software and liquid scintillation detectors of various sizes. During the measurement campaign in April 2012, four detectors $(7.62 \mathrm{~cm}$ x $7.62 \mathrm{~cm} \mathrm{EJ-309s)} \mathrm{were} \mathrm{used} \mathrm{with} \mathrm{a} \mathrm{4-channel} \mathrm{USB} \mathrm{digitizer}$ (CAEN DT5720) and a data-acquisition laptop, making the system portable.

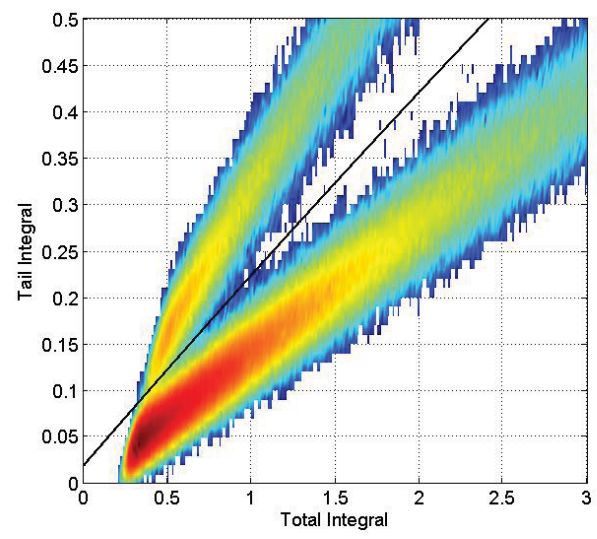

Fig. 2. Photon discrimination through PSD techniques at a $70 \mathrm{keVee}$ lightoutput threshold (approximately $650 \mathrm{keV}$ neutron energy deposited).

The liquid scintillators are sensitive to both neutrons and photons; therefore, both particle types create pulses that are digitized, and kept for data analysis. The digitizer has a 12-bit resolution (11 bits effective) and a 250-MHz sampling frequency which is sufficient for good off-line pulse-shape discrimination (PSD) to separate neutron events from photon events (Fig. 2). A thin lead shield $\left(0.2 \mathrm{~cm}\right.$ for $\mathrm{PuO}_{2}$ and $1 \mathrm{~cm}$ for MOX) was also present to decrease photon detection. The detectors were placed at $20 \mathrm{~cm}$ from the center of the source and were arranged in a small arc with approximately 30degrees between each detector. Fissile materials including $\mathrm{PuO}_{2}$ pellets and MOX powder were measured.

\section{B. Measurement-Data Analysis}

The first stages of the data analysis include photon rejection through a standard charge integration PSD method, shown in Fig. 2. After PSD, neutron data were analyzed both by determining the energy deposited in the detector by each neutron detection, as well as analyzing neutron multiplicity in short time windows $(\sim 100 \mathrm{~ns})$. This paper focuses on neutron coincidence and neutron pulse-height results for use in nuclear safeguards applications.

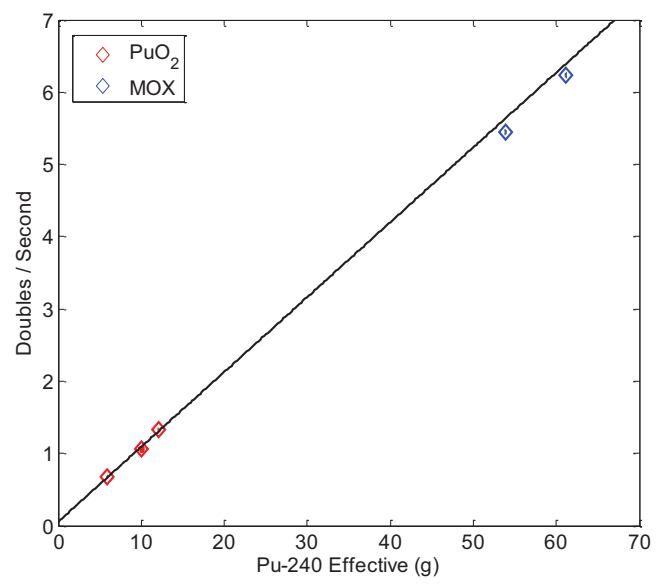

Fig. 3. Relationship between neutron doubles rate and plutonium-240 effective mass in configurations of $\mathrm{PuO}_{2}$ pellets and $\mathrm{MOX}$ powder.

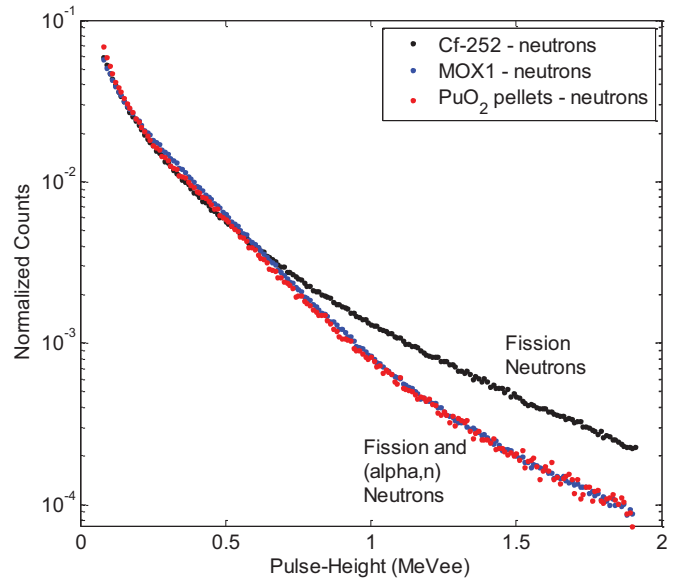

Fig. 4. Measured neutron pulse-height distributions (normalized to their integral) for Cf-252, $\mathrm{MOX}$, and $\mathrm{PuO}_{2}$ pellets.

The plutonium-mass sensitivity of the measurement system was studied via the measured doubles rates (neutron coincidence) for $\mathrm{PuO}_{2}$ and MOX samples, outlined in Table 1. The sensitivity of the passive measurement system can be characterized by the shape of the progression of neutron coincidence counts per gram of plutonium-240 effective mass (shown in Fig. 3). The relationship between the neutron coincidence rate and the $\mathrm{Pu}-240$ effective mass is linear across all of the $\mathrm{PuO}_{2}$ and $\mathrm{MOX}$ samples, and therefore the slope of this line can serve as the sensitivity metric. This metric is approximately 0.1 coincidence neutrons per second per gram for this bench-top system. These preliminary results bode well for a full fast-neutron detection system's ability to quantify plutonium mass from fast-neutron coincidence. Fig. 4 shows pulse-height distributions (PHDs) that shed light on the type of 
neutron source that is being measured, for example a plutonium metal sample will give different PHD results than a plutonium-oxide sample. This information can prove useful to tailor mass quantification equations to specific nuclear material types.

\section{Simulation AND MOdELING TOOLS FOR NUCLEAR SAFEGUARDS APPLICATIONS}

Several nuclear safeguards detection systems using organic scintillation detectors are being investigated using the MCNPX-PoliMi transport code and the MPPost postprocessing code [2]. MCNPX-PoliMi is ideal for designing detection systems, such as fast-neutron-multiplicity counters, due to its: capability of realistically simulating correlated source events, detailed particle interaction output, and incorporation of common SNM sources with accurately sampled energy, number of particles emitted, and their angle distributions [3]. MPPost processes the MCNPX-PoliMi data file into total measurement system response [3]. At UM, the measurement system design process is expedited with the UM parallelized version of MCNPX-PoliMi coupled with UM's advanced computing resources.

\section{A. Simulating Passive Systems with MCNPX- PoliMi/MPPost}

MCNPX-PoliMi was used to model the experimental geometry as well as the radiation sources. The complex neutron and photon sources present in the plutonium and oxygen containing materials were modeled based on built-in MCNPX-PoliMi sources: $\mathrm{Pu}-240$ and $\mathrm{Pu}-242$ spontaneous fissions and $\mathrm{Pu}-238, \mathrm{Pu}-239, \mathrm{Pu}-240$, and $\mathrm{Am}-241$ alpha-n reactions. The neutron emission contributions for both materials are shown in Fig. 5. In all of the configurations, detailed information was recorded for the four organic scintillation detectors. These data are then analyzed using MPPost to arrive at PHDs and fast-neutron multiplicity.

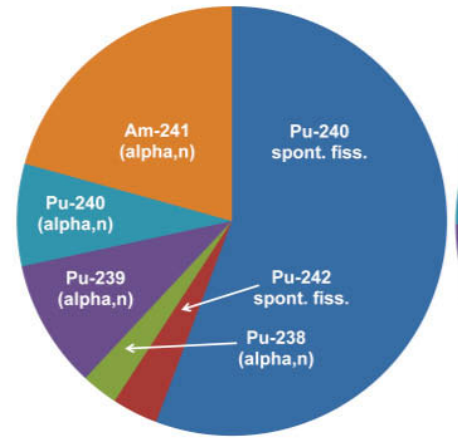

(a)

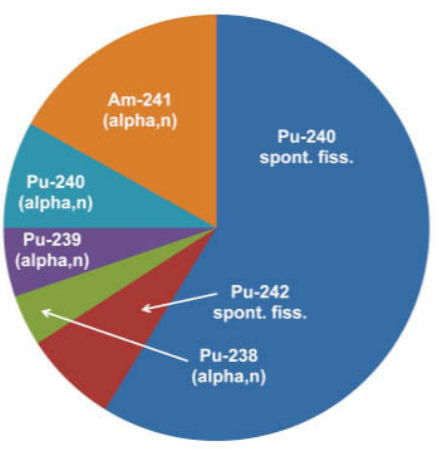

(b)
Fig. 5. The neutron-source contributions for the $\mathrm{PuO}_{2}$ pellets (a) and MOX (b) measured at the JRC facility.

\section{B. Validating MCNPX-PoliMi/MPPost}

Fig. 6 shows good agreements between the simulated and measured neutron PHDs from a $\mathrm{Cf}-252$ source. With a more complex neutron source $\left(\mathrm{PuO}_{2}\right.$ pellets described in Table 1 and Fig. 5a) good agreement is still observed for neutron doubles rates over a range of plutonium mass (shown in Fig.
7), the measurement result of primary concern for the design process. This 'small-size' system had promising Cf-252 absolute efficiencies of $4 \%$ for neutron singles and $0.06 \%$ for neutron doubles. This is promising considering any final system design would include many more detectors and cover much more solid angle.

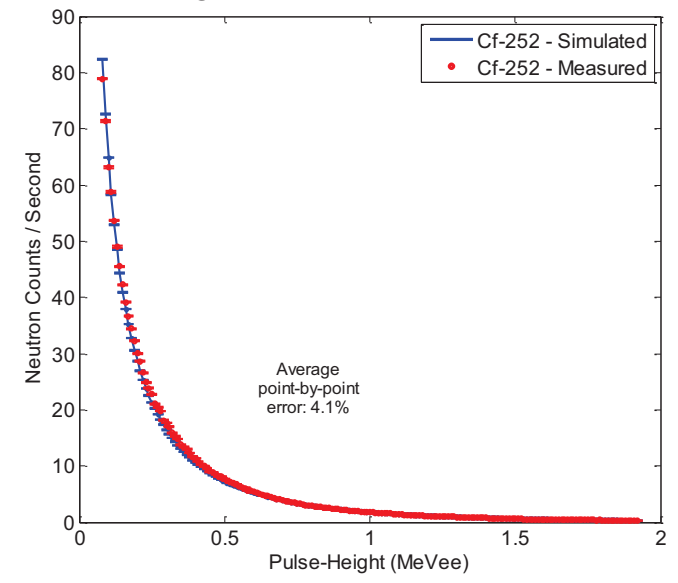

Fig. 6. Simulated and measured Cf-252 PHDs with an average point-bypoint agreement error of $4.1 \%$.

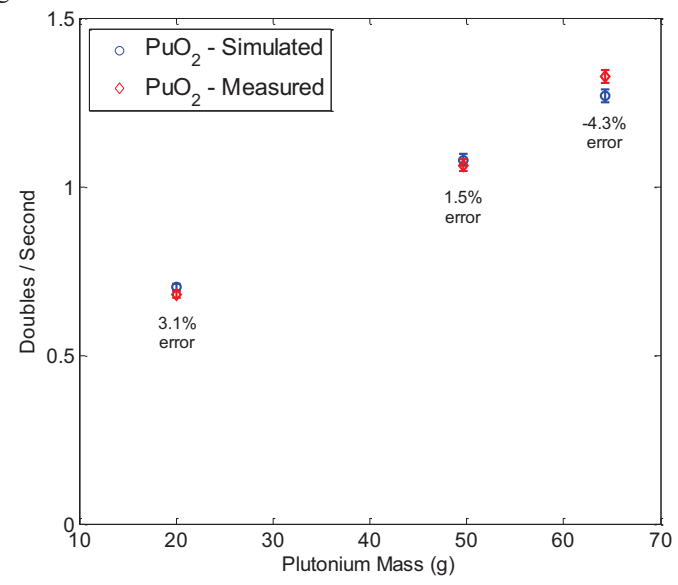

Fig. 7. Simulated and measured neutron doubles from three measurements of $\mathrm{PuO}_{2}$ pellets of increasing mass with statistical errors between 2 and $4 \%$.

\section{CONCLUSIONS AND FUTURE WORK}

Liquid scintillators appear to be a good candidate for a fastneutron-multiplicity counter. Fast-neutron doubles rates (from multiplicity) trend with plutonium-240 eff. mass in $\mathrm{PuO}_{2}$ and MOX samples (Fig. 4). Also, Neutron PHDs (Fig. 3) allow source type characterization (fission sources only versus fission and alpha-neutron sources, e.g. metal versus oxide) in liquid scintillation detectors. Lastly, the agreement (less than $5 \%$ error) between the fast-neutron measurement system's simulation and experimental campaigns validate the use of the MCNPX-PoliMi/MPPost package for designing a fastneutron-multiplicity counter.

Current simulation efforts are in progress to design a full fast-neutron-multiplicity counter. The design process utilizes the discussed system sensitivity as the metric to optimize and select the detector shape, size, number, and configuration. The goal is to design a system with high efficiency, high sensitivity, and minimal dependence on sample placement. An 
ideal detector design will not only perform accurately and efficiently, but also maintain a minimally intrusive geometry in terms of size and weight. With a list of candidate designs, bench-top experiments are being performed at UM to work towards a final configuration.

\section{ACKNOWLEDGMENT}

The University of Michigan's Detection for Nuclear Nonproliferation group thanks the staff PERLA laboratory staff at the Joint Research Centre in Ispra for their help making these benchmarking measurements possible.

\section{REFERENCES}

[1] E. Padovani, S. A. Pozzi, S. D. Clarke, E. C. Miller, "Introduction to MCNPX-PoliMi", Version 2.7, (2012)

[2] E.C. Miller, A. Poitrasson-Riviere, A. Enqvist, J.L. Dolan, S. Prasad, M.M. Bourne, K. Weinfurther, S.D. Clarke, M. Flaska, S.A. Pozzi, E. Padovani, J.K. Mattingly, "MCNPX-PoliMi Post-Processor (MPPost) Manual," Version 2.1.0, (2012).

[3] S. A. Pozzi, S. D. Clarke, W. Walsh, E. C. Miller, J.L. Dolan, M. Flaska, B. M. Wieger, A. Enqvist, E. Padovani, J. K. Mattingly, D. Chichester, and P. Peerani, "MCNPX-PoliMi for Nuclear Nonproliferation Applications," NIM-A, vol. 694, pp. 119-125 (Sept. 2012). 